\title{
Traumatic brain injury causes selective, CD74-dependent peripheral lymphocyte activation that exacerbates neurodegeneration
}

Richard P Tobin ${ }^{1}$, Sanjib Mukherjee ${ }^{1,2}$, Jessica M Kain 1,2, Susannah K Rogers ${ }^{3}$, Stephanie K Henderson ${ }^{4}$, Heather L Motal ${ }^{1}$, M Karen Newell Rogers ${ }^{1,4,5^{*}+}$ and Lee A Shapiro ${ }^{1,2,4 \dagger}$

\begin{abstract}
Introduction: Traumatic brain injury (TBI), a significant cause of death and disability, causes, as in any injury, an acute, innate immune response. A key component in the transition between innate and adaptive immunity is the processing and presentation of antigen by professional antigen presenting cells (APCs). Whether an adaptive immune response to brain injury is beneficial or detrimental is not known. Current efforts to understand the contribution of the immune system after TBI have focused on neuroinflammation and brain-infiltrating immune cells. Here, we characterize and target TBI-induced expansion of peripheral immune cells that may act as potential APCs. Because MHC Class II-associated invariant peptide (CLIP) is important for antigen processing and presentation, we engineered a competitive antagonist (CAP) for CLIP, and tested the hypothesis that peptide competition could reverse or prevent neurodegeneration after TBI.

Results: We show that after fluid percussion injury (FPI), peripheral splenic lymphocytes, including CD4+ and CD8+ T cells, regulatory $T$ cells (Tregs), and $\gamma \delta T$ cells, are increased in number within 24 hours after FPI. These increases were reversed by CAP treatment and this antagonism of CLIP also reduced neuroinflammation and neurodegeneration after TBI. Using a mouse deficient for the precursor of CLIP, CD74, we observed decreased peripheral lymphocyte activation, decreased neurodegeneration, and a significantly smaller lesion size following TBI.
\end{abstract}

Conclusion: Taken together, the data support the hypothesis that neurodegeneration following TBI is dependent upon antigen processing and presentation that requires CD74.

Keywords: TBI, FPI, Fluid percussion injury, CD74, Neuroinflammation

\section{Introduction}

Traumatic brain injury (TBI) is a leading cause of death and disability in the United States and throughout the world [1-3]. To date, there are no effective treatments to prevent or reverse damage after TBI. Due to the direct impact on the brain, efforts at diagnosis and treatment of TBI have been primarily focused on the central nervous system (CNS). However, it is now well established that peripheral immune mechanisms are involved in the pathology associated with TBI [4-7]. The paradigm that

\footnotetext{
*Correspondence: newellrogers@tamhsc.edu

${ }^{\dagger}$ Equal contributors

'Department of Surgery, Texas A\&M University Health Science Center, Temple, TX, USA

${ }^{4}$ Department of Surgery, Baylor Scott and White Health, Temple, TX, USA

Full list of author information is available at the end of the article
}

has been widely accepted is that the blood brain barrier (BBB) serves as the primary obstacle to the recruitment of leukocytes that could otherwise cause harm to the brain $[7,8]$. However, after TBI, there is BBB breakdown and one result is the passage of peripheral immune cells into the CNS [7].

In general, an immune response will begin with an innate and immediate response to injury that includes activation and migration of neutrophils, dendritic cells, macrophages (including microglial cells in the CNS), as well as other less defined cellular and molecular components [9-11]. The innate immune response is typically followed by the antigen-specific, adaptive immune response. The transition from an innate to an adaptive 
response is dependent upon successful antigen processing and presentation, by professional antigen presenting cells (APCs), to antigen-specific T lymphocytes $[12,13]$. How, or if, damage to the brain, often described as an "immune-privileged" site [14], elicits an innate, and the transition to, an adaptive immune response from peripheral immune mediators to the CNS, is not known. Evidence for the involvement of antigen processing and presentation in the CNS is provided by the study of Slavin et al. [15]. This study demonstrated that mice lacking the machinery for processing and presentation of full-length myelin basic protein did not develop experimental autoimmune encephalomyelitis (EAE), a mouse model for Multiple Sclerosis (MS). However, when these same mice were exposed to peptides derived from full-length myelin basic protein, the mice became susceptible to EAE. Interestingly, Ling et al. demonstrated that TBI results in recruitment of antigen-specific CD8+ T cells, only in the presence of the antigen for which they are specific [16]. Furthermore, in a model of optic neuritis, the acute immune response leads to an adaptive, specific, anti-brain response [17-19]. Conflicting reports suggest that this effect could be either harmful or helpful to recovery $[5,6]$. Regardless of their role in the outcome, these findings provide evidence for an adaptive immune response in the brain after TBI.

Efficient antigen processing and presentation via the major histocompatibility complex II (MHCII) pathway in APCs, including macrophages, microglia, dendritic cells, B cells, and $\gamma \delta$ T cells, facilitates the transition between innate and adaptive immunity [13,20-22]. Antigen processing and presentation by these professional APCs involves invariant chain (CD74). CD74 serves as a chaperone for MHCII until CD74 is proteolytically cleaved into Class II invariant peptides (CLIP) that fill the antigen-binding groove of MHCII molecules [12,23]. Replacement of CLIP by antigenic peptides and T cell recognition of antigenic peptide in MHCII is the first step in $\mathrm{T}$ cell activation, and the first event in a $\mathrm{T}$ cell-mediated adaptive immune response $[13,20,24]$. This vital step of peptide exchange between innate and adaptive immunity has not been elucidated following TBI. Critical to this process is the cleavage of full-length CD74 into CLIP, the loading of CLIP into the antigenic binding groove of MHCII, followed by the replacement of CLIP with antigenic peptides [23]. Therefore, we engineered and synthesized a peptide that competitively antagonizes the antigenbinding site of MHCII, to determine if antagonism of CLIP/antigenic peptide exchange might prevent or reverse the detrimental effects of TBI [25]. Thus, this study was designed to determine if exchanging CLIP for antigenic peptide is important in secondary neurodegeneration following TBI.

\section{Materials and methods}

Treatment with competitive antagonist peptide (CAP)

CAP was synthesized as previously described [25]. Briefly, using computational design, we identified a 9mer peptide, with 8 total amino acid flanking regions, that by, peptide binding analysis software (MHCPred and netMHC), was predicted to have a higher binding constant than the invariant peptide CLIP for the peptide-binding groove of known MHCII alleles. CAP was synthesized by Elim Biopharmaceuticals. The mice were injected intraperitoneally (i.p.) $(1 \mathrm{mg} / \mathrm{kg})$ with CAP. CAP was initially dissolved at $5 \mathrm{mg} / \mathrm{mL}$ in dimethyl sulfoxide (DMSO), after which $5 \mu \mathrm{l}$ of CAP dissolved in DMSO was further diluted with $195 \mu \mathrm{l}$ of sterile saline and injected (I.P.). In vehicle injected mice, an injection containing $5 \mu \mathrm{l}$ DMSO dissolved in $195 \mu \mathrm{l}$ of sterile saline.

\section{Animals}

6-week-old male C57BL/6 J $(\mathrm{N}=16)$ were purchased from Jackson Labs. Invariant chain deficient (CD74 ${ }^{\text {Def }}$ ) mice (C57BL/6 background; $\mathrm{N}=16$ ) were purchased from Jackson Labs and bred at the Scott and White Healthcare animal facility to maintain homozygous CD74 deficiency. Because littermates are not available to compare with the CD74 ${ }^{\text {Def }}$ mice (as they are bred to homozygosity), we have used age and sex matched C57BL/6 mice (the background strain for the mice). All mouse care, housing, and experimental procedures were approved by the Baylor Scott and White Healthcare Institutional Animal Care and Use Committee, reference number 2013-021. Wild type mice were divided into four groups for flow cytometry and multiplexing and sacrificed at $24 \mathrm{hrs}$ after TBI. These four groups were: sham + vehicle $(\mathrm{N}=6)$; sham + CAP $(\mathrm{N}=5) ; \mathrm{FPI}+$ vehicle $(\mathrm{N}=7 \mathrm{WT}) ; \mathrm{FPI}+\mathrm{CAP}(\mathrm{N}=6)$. For the $\mathrm{CD} 74^{\text {Def }}$ mice, two groups were used. These two groups were: Sham $\left(\mathrm{N}=7 \mathrm{CD} 74^{\text {Def }}\right)$ and FPI $\left(\mathrm{N}=6 \mathrm{CD} 74^{\text {Def }}\right)$. In addition, we used separate mice for histological studies and these mice were sacrificed 3 days after TBI $(\mathrm{N}=6 \mathrm{WT}$ sham + vehicle, $5 \mathrm{FPI}+\mathrm{CAP}$, and $\left.5 \mathrm{CD} 74^{\mathrm{Def}}\right)$. The three day post-TBI time point was selected because our previous study indicated that this is when the peak of TBIinduced neurodegeneration was observed [26].

\section{Fluid percussion injury (FPI)}

FPI was induced as previously described [26]. Briefly, mice were anesthetized with isoflurane, prepped, cleaned, and shaved, then put into a stereotactic instrument (Stoelting, Inc.). A $2 \mathrm{~mm}$ craniotomy was made over the left parietal cortex making sure to keep the dura intact. The female end of a luer lock syringe was cemented over the craniotomy and attached to the fluid percussion apparatus. A 12-16 ms FPI was delivered at a pressure of $\sim 1.4$ 1.6 atm. Sham mice received identical treatment except 
the pressure pulse was never delivered. After injury or sham, suture was used to close the scalp over the wound and mice were returned to their home cage resting on a heating pad. Mice were monitored to ensure that they resumed walking, feeding, drinking and grooming behavior.

\section{Flow cytometry}

Mice were sacrificed and spleens were removed. The tissues were dissociated, single cell suspensions prepared, and red blood cells were lysed using GEY'S buffer [27]. The dissociated splenocytes were treated with FC Block (BD Bioscience) and then stained with the following antibodies; CD3, CD4, CD8, CD25, MHCII (clone M5/114.15.1), B220, $\gamma \delta$-TCR (BD Bioscience), and CLIP (15G4, Santa Cruz Biotechnology). For regulatory $\mathrm{T}$ cell (Treg) staining we used the eBioscience mouse regulatory $\mathrm{T}$ cell staining Kit according to the manufacturer's directions. Live cells were assessed using the Life Technologies LIVE/DEAD ${ }^{\bullet}$ Fixable Aqua Dead Cell Stain Kit according to the manufacturer's directions. Splenocytes were analyzed using a BD FACS Canto II flow cytometer and data were analyzed using FlowJo software (TreeStar Inc.). See Additional file 1: Figure S1 for gating strategy.

\section{FluoroJade C method}

Fluorojade $\mathrm{C}$ staining took place as previously described [26]. Briefly, every 6th 50 um section was analyzed within the margins of the lesion. This corresponded to 5-7 sections per mouse. Sections were mounted onto gelatin-coated slides and FluoroJade $C$ histology was performed as previously described [26]. The slides were allowed to air dry, after which cover slips were applied using Vectashield with DAPI (Vector Labs). Prior to analysis, slides were coded to ensure that the reviewer was blind to the condition of the mice. Sections were then visualized using an Olympus IX81 (Olympus Inc., Tokyo, Japan) inverted microscope equipped to visualize FITC. We used the following Olympus UplanS objectives to capture the images: $10 \times \mathrm{NA0.40} \infty / 0.17$ FN26.5; $20 \times$ nA0.75 $\infty / 0.17$ FN26.5; 40× NA0.75 $\infty / 0.17$ FN26.5. Images were captured using the FV1000 and the Fluoview software (V. 1.7.1.0). Images were saved as 24 Bit Tiff files and brightness and contrast were adjusted using Adobe Photoshop (V. 12.0). For quantitative analysis of Fluorojade $\mathrm{C}$ labeling, the peri-lesion area was traced and $400 \mu \mathrm{m}^{2}$ grids were randomly placed throughout the traced area. All cells which fell within the grid, or contacted the left and upper borders of the grid were included in the counts, whereas those that touched the right or lower border were excluded. The peri-lesion area consisted of $+/-1 \mathrm{~mm}$ anterior/posterior (AP) from the injury focus $(\mathrm{AP}+1.5 \mathrm{~mm}$; medio-lateral: $1.2 \mathrm{~mm})$.

\section{Measurement of lesion size}

Superficial lesion size was measured using a digital caliper (Mitutoyo $150 \mathrm{~mm}, .01 \mathrm{~mm}$ accuracy) as previously described $[28,29]$. In order to confirm superficial lesion measurements made with the digital caliper, we assessed the margins of the lesion in the anterior posterior plane, and calculated the expanse of the lesion using a mouse atlas [30]. Initially, every 6th $50 \mu \mathrm{m}$ Nissl stained section was used for determination. However, in some cases, it was necessary to examine serial sections in which Fluorojade $\mathrm{C}$ (or other stains not reported in this manuscript) was stained, in order to confirm that we had accurately accounted for the most anterior and posterior margins of the lesion. It should be noted that considering the margin of error of the digital caliper $(100 \mu \mathrm{m})$ and the distance between brain slices in the mouse atlas (100$140 \mu \mathrm{m})$, all measurements were within the margin of error of these 2 sources.

\section{Cytokine analysis}

Fresh, non-perfused tissue was isolated from the ipsilateral cortex including the lesion area and flash frozen in liquid nitrogen. Frozen tissue was homogenized following the manufacturer's instructions (Milliplex MAP kit, Millipore). Protein was estimated with a Bradford assay and similar concentrations were made such that $25 \mu \mathrm{l}$ of homogenate was added to $25 \mu \mathrm{l}$ of assay buffer. Then, $25 \mu \mathrm{l}$ of magnetic beads coated with specific antibodies (MCYTOMAG70 K-PMX, Milliplex MAP Kit, Millipore) was added to this solution and the reaction was incubated at $4^{\circ} \mathrm{C}$ for 24 hours. Next, the beads were washed and incubated with $25 \mu \mathrm{l}$ of biotinylated detection antibody at room temperature (RT) for 2 hours. Finally, $25 \mu \mathrm{l}$ of Streptavidin-Phycoerythrin conjugate compound was added and incubated for $30 \mathrm{~min}$ at RT. The beads were then washed and incubated with $150 \mu \mathrm{l}$ of sheath fluid for $5 \mathrm{~min}$ at RT. The concentration of the analytes is then determined by Bio-Plex Manager software version 5.0. The assays were run in triplicate to confirm the results. Analytes were normalized to total protein concentration. In cases where the cytokines in the brain tissue of three or more mice for a group were below the detection threshold, the data were not presented in the graphs.

\section{Statistical analysis}

Statistical analysis was performed using GraphPad Prism 6 (GraphPad Software Inc.). For comparisons between splenocytes from C57BL/6 J and CD74 ${ }^{\text {Def }}$, a $t$-test was used with a significance cut-off of $\mathrm{P}<0.05$. For all other analysis, repeated measures ANOVA was used with post-hoc planned comparisons using Dunnett's correction factor. 


\section{Results}

Expansion of peripheral lymphocytes following Fluid Percussion Injury (FPI)

To characterize the changes in peripheral immune cells following TBI, splenic lymphocytes were harvested at 24 hours after FPI and analyzed using flow cytometry. The results showed that the overall splenic cellularity was significantly increased $(\mathrm{P}<0.017)$ in FPI-treated mice compared to sham-treated controls and this effect was prevented by administration of competitive antagonist peptide (CAP) (Figure 1A). While there was a trend toward increased B220+ B cells (Figure 1B), there was a significant $(P<0.03)$ reduction in $B$ cell numbers following CAP treatment after FPI (Figure 1B). In addition, there was a significant $(\mathrm{P}<0.001)$ FPI-induced increase in the total number of $\mathrm{CD} 3+\mathrm{T}$ cells that was significantly $(\mathrm{P}<0.001)$ reduced by $\mathrm{CAP}$ treatment after FPI (Figure 1C). Specifically, CD4+ T cells were also significantly increased in number $(\mathrm{P}<0.014)$ and their numbers were significantly reduced $(\mathrm{P}<0.018)$ following CAP treatment (Figure 1D). There were also significant increases in Tregs $(\mathrm{P}<0.018)$, as defined by $\mathrm{CD} 3+, \mathrm{CD} 4+$, high CD25 and expression of the transcription factor FoxP3 (Figure 1E), $\gamma \delta \mathrm{T}$ cells $(\mathrm{P}<0.0 .14)$; (Figure $1 \mathrm{~F})$ and CD8+ T cells $(\mathrm{P}<0.02)$; (Figure $1 \mathrm{G})$, all of which were significantly reduced by treatment with CAP (Figures $1 \mathrm{E}-\mathrm{G}$ ). In contrast, there was no significant increase in the numbers of conventional activated CD4+ T cells, nor was there a significant reduction of these cells following CAP treatment (Figure 1H).

\section{FPI-induced changes in components of antigen} presentation by peripheral lymphocytes

A hallmark of the transition between innate and adaptive (specific) immunity is successful antigen processing and presentation. Antigen processing requires proteolytic cleavage of invariant chain (CD74) into peptides known as class II invariant peptide (CLIP). CLIP functions as a "placeholder" for antigen specific binding to the peptidebinding groove of MHCII molecules. B cells and $\gamma \delta$ T cells

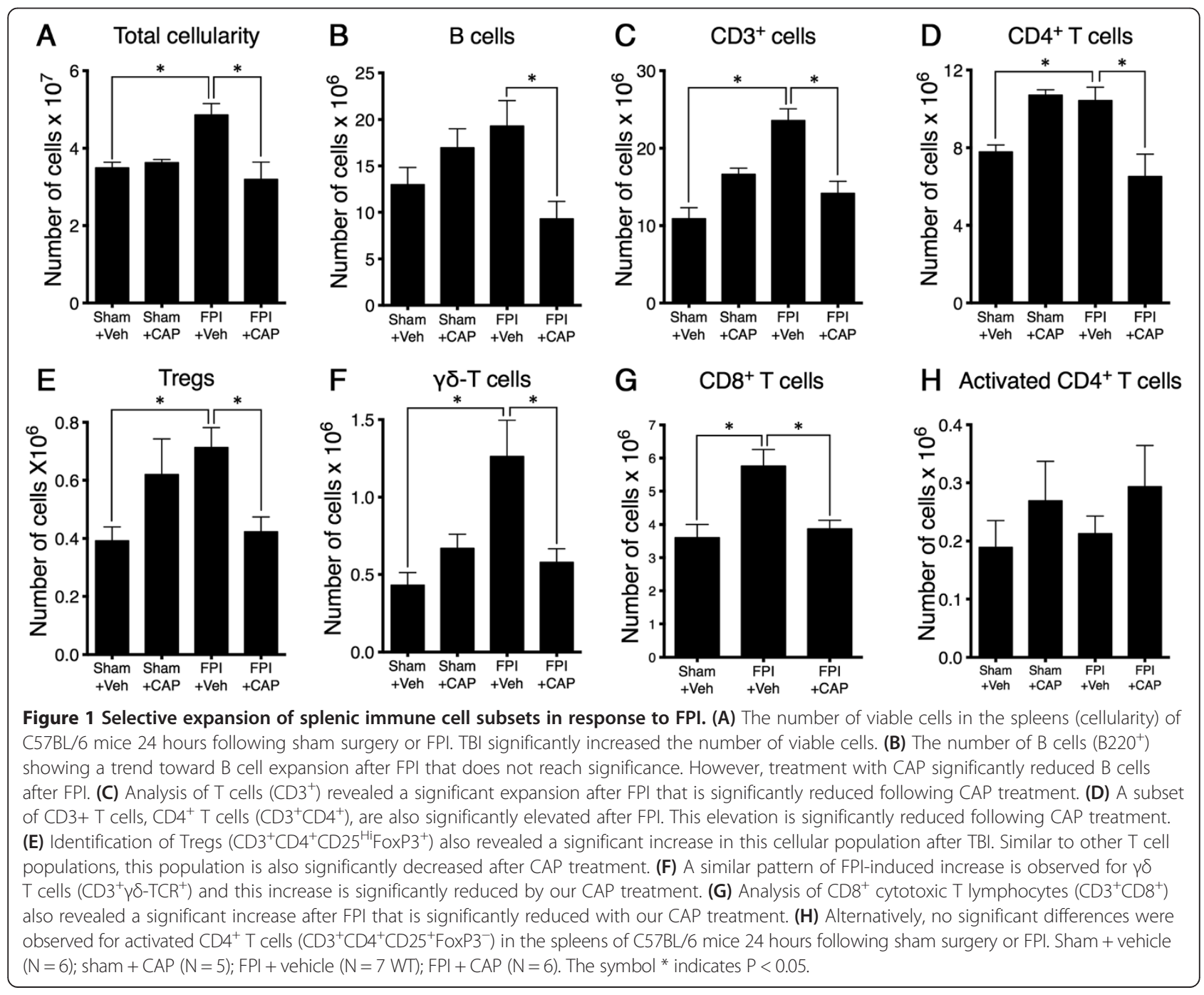


are among cells that are well characterized as professional APCs [31,22]. Therefore, we examined the effects of FPI on CLIP and MHCII from peripheral lymphocytes. We observed a significant decrease in cell surface CLIP on B cells (Figure 2A). Conversely, we observed no corresponding changes in the cell surface levels of MHC II on B cells (Figure 2B). In contrast to CLIP on B cells, we observed no significant differences in the level of CLIP per $\gamma \delta \mathrm{T}$ cells (Figure 2C), but a significant decrease in MHCII on $\gamma \delta$ T cells (Figure 2D). These results suggest possible FPIinduced alterations to molecular machinery involved in antigen processing and presentation.

To test the effect of our peptide on influencing the transition between innate and adaptive immunity, we examined CLIP and MHCII expression on B cells and $\gamma \delta \mathrm{T}$ cells in mice administered CAP 20 minutes after FPI. We examined these two cell types because they are known to express $\mathrm{MHCII}$ and present antigens. We observed no significant differences in CLIP expression on the cell surface of B220+ B cells (Additional file 2: Figure S2) following CAP administration after FPI. These results suggest that of these 2 potential APCs (B cells or $\gamma \delta$ T cells), the data are more consistent with B cells functioning as APCs in response to $\mathrm{TBI}$. In contrast, the data are less suggestive of $\gamma \delta \mathrm{T}$ cells serving as APCs based on reduced MHCII (Figure 2D) and therefore a higher ratio of CLIP to MHCII on $\gamma \delta$ T cells.

\section{Absence of FPI-induced lymphocyte expansion in invariant chain deficient mice}

Because we observed changes in CLIP expression resulting from FPI, we sought to determine the significance of CLIP involvement in immune modulation by FPI, by using mice deficient in invariant chain, CD74. CD74, which is required for processing and presentation of full-length proteins $[18,15]$, can serve as a survival factor and can function as a growth promoting receptor. In support of the notion that CD74 is required for lymphocyte expansion following FPI, mice lacking CD74 exhibited no net change in spleen cellularity 24 hours following FPI (Figure 3A). Alternatively, wild type mice exhibit a significant expansion of lymphocytes in the spleen at $24 \mathrm{hrs}$ after FPI (Figure 1A). Furthermore, there were no changes in numbers or activation state of any of the subsets of $\mathrm{B}$ or $\mathrm{T}$ cells (Figure $3 \mathrm{~B}$ and $\mathrm{C}$, respectively).

\section{CLIP antagonism or invariant chain deficiency modifies} the cytokine profile in the CNS following FPI

Our previous work has identified numerous changes in cytokines and chemokines in the CNS following an FPI [32]. Because CAP reduced the number of activated splenocytes following FPI, and activated splenocytes are a potential source of cytokines and chemokines, we sought to determine if CLIP antagonism using CAP modifies the cytokine milieu in the CNS. In order to address this, we screened for multiple cytokines, using a Multiplex cytokine assay system (MCYTOMAG-70 K-PMX). The results showed that CAP treatment significantly reduced IL15, IL13, IL10 and monocyte chemoattractant protein (MCP1), also known as CCL2 (Figure 4).

Because the absence of invariant chain in the CD74 def mice resulted in no splenocyte activation after FPI, we similarly sought to examine the cytokine and chemokine expression in the brains of these KO mice after FPI. We observed significantly less TBI-induced expression of IFN $\gamma$, TNF $\alpha$, regulated on activation normal T cell expressed and secreted (RANTES; AKA CCL5), macrophage inflammatory protein $1 \beta$ (MIP1 $\beta$; AKA CCL4) and IL-13, in the CNS of $\mathrm{CD} 74^{\text {def }}$ mice when compared to $\mathrm{C} 57 \mathrm{BL} / 6$
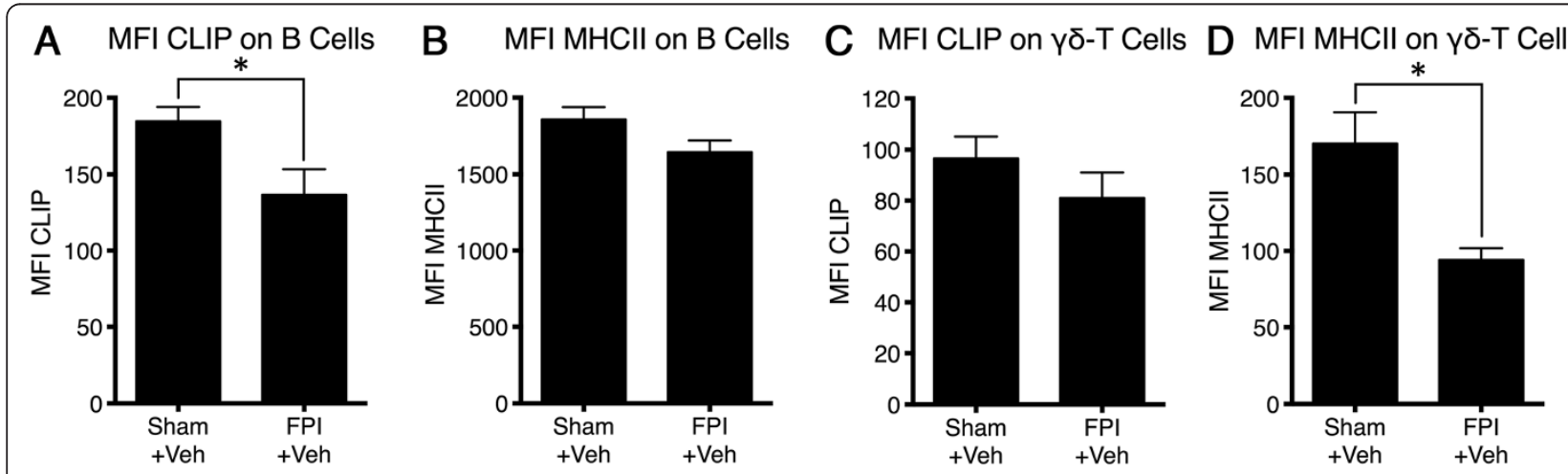

Figure 2 Differential expression of $\mathbf{M H C I I}$ and CLIP on B and $\boldsymbol{\gamma} \delta$ T cells in response to FPI. (A) The mean fluorescence intensity (MFI), indicative of the level of cell surface expression of CLIP is significantly reduced on the surface of B cells at 24 hours after FPI. This reduction in CLIP likely reflects replacement of CLIP by other peptides. (B) Consistent with this interpretation, no corresponding significant decrease in the MFI of $\mathrm{MHCll}$ on the surface of B cells was observed at 24 hours after FPI. (C) In contrast, the MFI of CLIP on the surface of $\gamma \delta \mathrm{T}$ cells remained unchanged, (D) while the level of MHCII significantly decreased at 24 hours after FPI. Taken together, these data suggest that B cells are a likely candidate as APCs after FPI. Sham + vehicle $(N=6)$; FPI + vehicle $(N=7)$. The symbol * indicates $P<0.05$. 


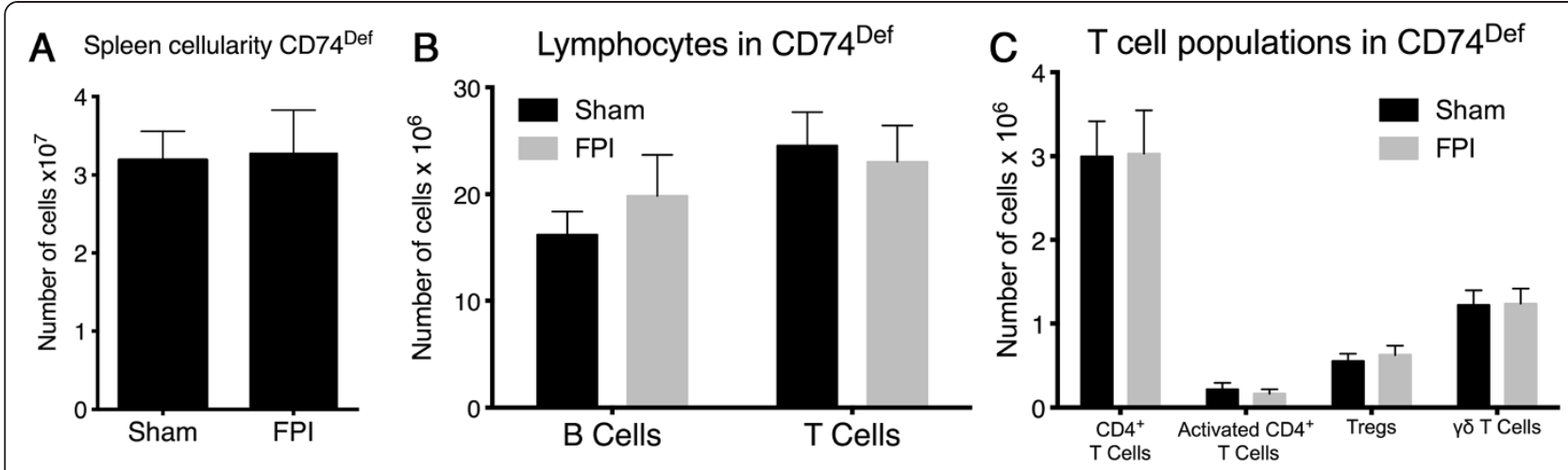

Figure 3 FPI-induced alterations in peripheral lymphocytes require CD74. (A) The number of viable cells in the spleens of CD74 ${ }^{\text {Def }}$ mice is unchanged at 24 hours following sham surgery or FPI. (B) Similarly, the number of B Cells $\left(B 220^{+}\right)$and T cells $\left(C D 3^{+}\right)$in the spleens of CD74 ${ }^{D e f}$ mice are not significantly different at 24 hours after sham surgery or FPI. (C) Analysis of specific populations of T cells also revelaed no significant differences at 24 hours after sham surgery or FPI. For these experiments we used 5 sham surgery mice and 6 FPI treated mice. The symbol * indicates $P<0.05$.

mice (which are the genetic background of these mice) (Figure 5).

\section{Invariant chain deficiency or CLIP antagonism confers neuroprotection from FPI}

To determine the contribution of CD74 to FPI-induced neurodegeneration, we examined FluoroJade $\mathrm{C}$ labeling at 3 days after FPI in WT and CD74 ${ }^{\text {Def }}$ mice. The 3 day time point was selected because we previously demonstrated that this was the peak of neurodegeneration following FPI [26]. The results demonstrate that relative to vehicle treatment (Figure 6A), treatment with CAP significantly reduced the number of FluoroJade C-labeled cells in the ipsilateral cortex (Figure 6B). Similarly, mice deficient for CD74 (Figure 6C) had decreased FluoroJade Clabeling compared to vehicle treatment (Figure 6A). In addition, Analysis of the overall lesion size revealed that both, CAP treated and CD74 ${ }^{\text {def }}$ mice had a significantly smaller lesion size compared to WT mice (Figure 6D). Quantitation of Fluorojade-staining in the brain following CAP administration and in CD74 $4^{\text {def }}$ mice, demonstrated a significant reduction in degenerating cells (Figure 6E).

\section{Discussion}

This study is the first to demonstrate a putative role for invariant chain CD74 in CNS damage following brain injury. The data support targeting the transition between innate and adaptive immunity in the periphery as a potential therapeutic approach for TBI. Alterations to peripheral immune cell populations include an increase in cellularity in the spleen, comprised of increased B cells, CD3+ T cells, CD4+T cells, CD8+ T cells, Tregs and $\gamma \delta$ $\mathrm{T}$ cells. Further, we found that TBI induces a decrease in cell surface CLIP on B220+ B cells, and a decrease in MHCII on activated $\gamma \delta$ T cells, suggesting that B cells may be the dominant APC after TBI. We also determined by using $\mathrm{CD} 74^{\text {Def }}$ mice, that these changes were invariant chain dependent.

The fact that CLIP antagonism and CD74 ${ }^{\text {Def }}$ mice have a reduced lesion size and decreased neurodegeneration after TBI, supports the involvement of CLIP and/or CD74 in mediating damage in the CNS. Both CD74 and its products, including CLIP, are widely accepted as central players in antigen processing and presentation, and are central to the transition between innate and adaptive
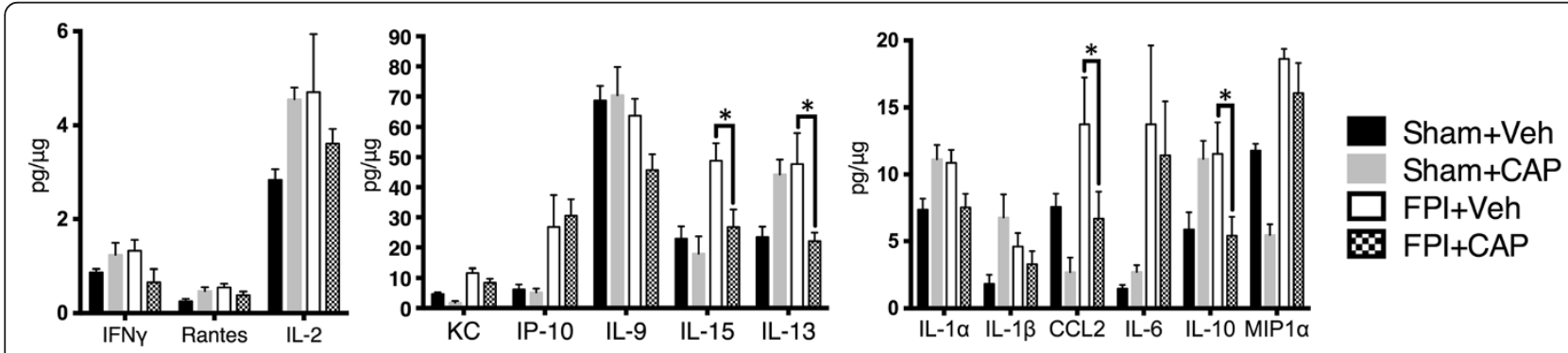

Figure 4 CAP decreases expression of pro-inflammatory cytokines in the brain following FPI. Multiplex analysis of cytokines in tissue from the ipsilateral cortex of C57BL/6 mice following FPI. Note the significant reductions in IL-13, IL-15, CCL2 (MCP1), and IL-10. The reduction of this subset of chemokines and cytokines is consistent with the reduction in cells capable of producing these inflammatory cytokines. Sham + vehicle $(N=4) ;$ sham + CAP $(N=4) ; F P I+$ vehicle $(N=4) ; F P I+C A P(N=4)$. The symbol * indicates $P<0.05$. 


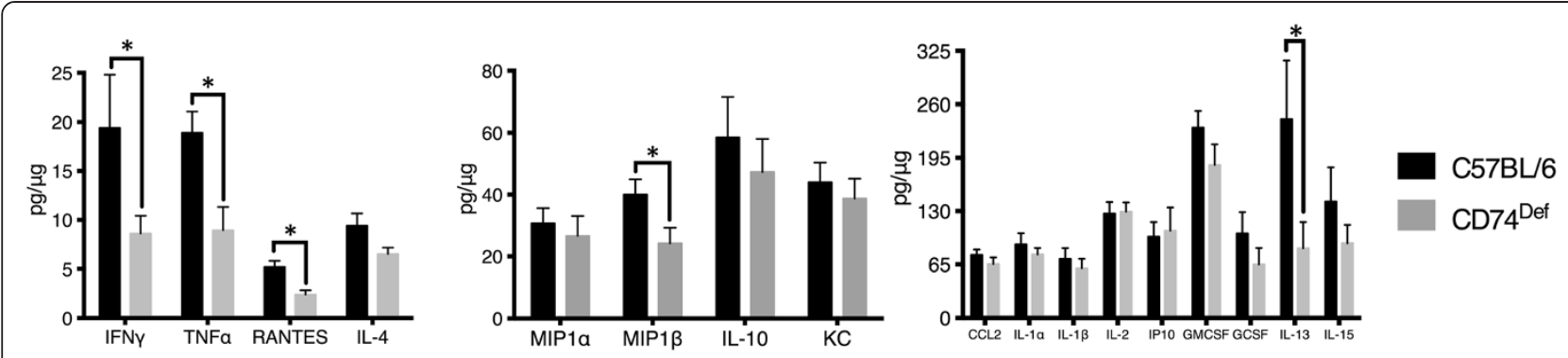

Figure $5 \mathrm{CD} 74^{\text {Def }}$ mice are resistant to FPI-induced increases in pro-inflammatory cytokines in the brain following TBI. Multiplex analysis of cytokine expression in tissue from the ipsilateral cortex, comparing the cytokine response to FPI in wild type C57BL/6 mice to CD74 ${ }^{\text {Def }}$ mice. The results demonstrate a significant decrease in the levels of TNFa, IFNY, RANTES, MIP1 $\beta$, and IL-13 in tissue from the ipsilateral cortex of CD74 ${ }^{\text {Def }}$ mice compared to C57BL/6 mice, measured at 24 hours post FPI. The reduction in this group of cytokines in the CD74 ${ }^{\text {Def }}$ mice, implicate CD74 in their production following FPI. For these experiments we used five C57BL/6 and six CD74 ${ }^{\text {Def }}$. The symbol * indicates $\mathrm{P}<0.05$.

immunity [12]. The neuroprotection observed in this study supports the involvement of antigen processing and presentation in the exacerbation of brain injury $[17,18,33,34]$. While it is clear that CD74 is involved in the damage to the CNS following TBI, several different functions have also been attributed to CD74. These include: survival, signal transduction through cell surface CD74, binding to pro-inflammatory macrophage inhibitory factor (MIF), along with its well-established role in antigen processing and presentation [35-38]. Based on the current findings, it is not possible to exclude any of these functions of CD74 in mediating neurodegeneration after TBI.

To further elucidate the putative role of CD74 in TBI, we engineered a peptide that competitively antagonizes the proteolytic product of CD74, CLIP [25]. We determined that administration of this potentially therapeutic peptide 20 minutes after TBI prevented the increase in splenic cellularity in all observed peripheral immune cell populations with the exception of activated conventional

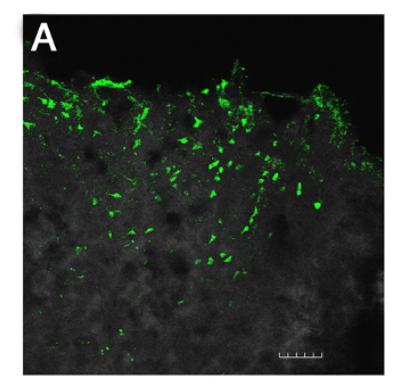

$\mathrm{D}$

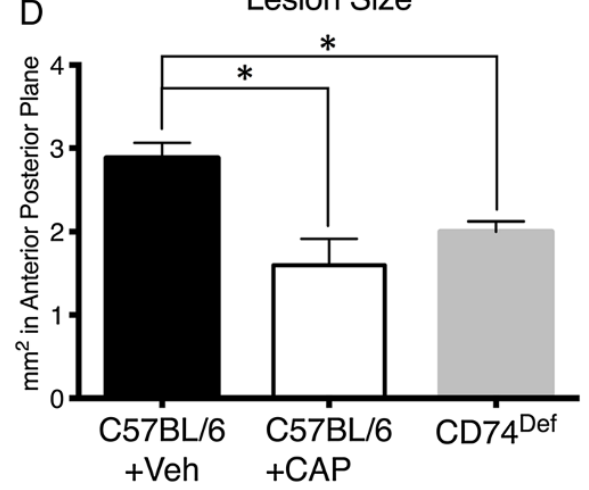

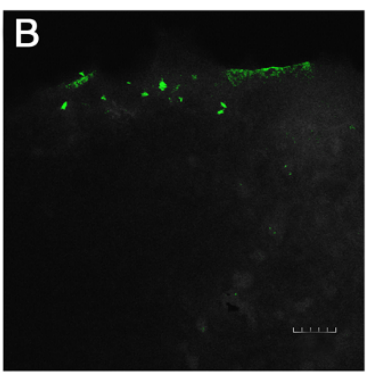

E

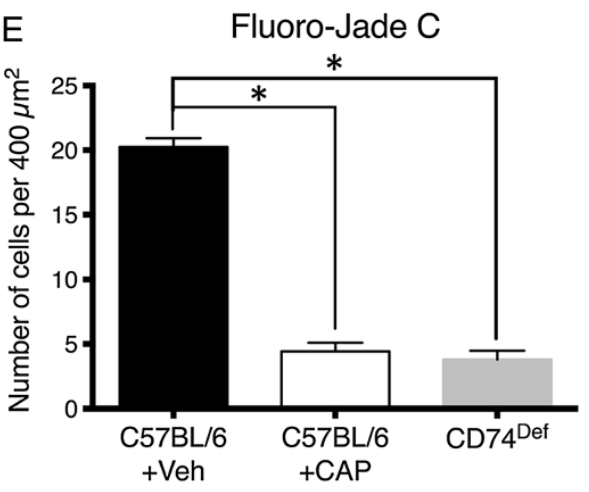

Figure 6 CAP treatment after FPI reduces neurodegeneration and lesion size. (A-C) Fluoro Jade $C$ labeling at 3 days after FPI. In vehicle treated mice (A), significantly more degenerating neurons are observed compared to mice treated with CAP after FPI (B) and CD74 def mice (C). (D) Analysis of the size of the lesion at 3 days after FPI revealed that CAP treatment and CD74 ${ }^{\text {Def }}$ mice had a significantly smaller lesion in the anterior/posterior plane, compared to sham mice. (E) Quantitative analysis of the number of FluorJade C-labeled cells showed significantly less $\left(\mathrm{P}<.01\right.$ for both) labeled-cells in the CAP treated and CD74 ${ }^{\text {Def }}$ mice. For this analysis, we used 6 shams, 5 CAP and 5 CD74 ${ }^{\text {Def }}$ mice. Scale bars in A-C $=50 \mu \mathrm{m}$. The symbol * indicates $P<0.01$. 
CD4+ T cells. Most importantly, administration of this peptide was neuroprotective following TBI, supporting the notion that neuronal damage involves the transition between innate and adaptive immunity.

The transition between innate and adaptive immunity can be regulated by several subsets of activated, non-conventional $T$ cells, including Tregs and $\gamma \delta T$ cells. While Tregs have been well described to dampen antigen-specific responses in the periphery, their role in the CNS is controversial [39-41]. Moalem et al. has proposed that following injury to the nervous system, Tregs may be detrimental, because the antigen specific $T$ cell response that they limit may be protective $[18,19,33]$. Alternatively, some investigators have proposed protective effects of Tregs following cerebral insults [40-43]. It is pertinent to note that two distinct subsets of Tregs have been characterized, natural Tregs and inducible Tregs $[44,45]$. Therefore, it is possible that one population of Tregs is beneficial and the other is detrimental. Future studies are needed to examine this possibility.

The current results also demonstrated a significant expansion of $\gamma \delta$ T cells after TBI that could be reversed with CAP treatment. Freedman and colleagues previously demonstrated the presence of $\gamma \delta$ T cells in the cerebral spinal fluid of patients with multiple sclerosis [46]. Additionally, it has previously been shown that these $\gamma \delta$ T cells could attack oligodendrocytes, indicating the potential for a destructive response by $\gamma \delta$ T cells in the CNS [46,47]. Our data showing a reduced MHCII expression by $\gamma \delta$ T cells after FPI argues for reduced capability for antigen presentation and processing. This reduced ability for antigen presentation and processing could prevent an effective transition from innate to adaptive immunity. Such prevention of an effective transition to adaptive immunity could undermine what would otherwise be protective autoimmunity as previously suggested $[18,33]$.

Similar to $\gamma \delta$ T cells, B cells are also well described as antigen specific, capable of producing antibodies, and as exquisitely efficient professional antigen presenting cells [21,22]. Our finding that after FPI, B cells have significantly reduced CLIP, but not significantly reduced MHCII, suggests that these cells have a higher likelihood for antigen presentation after TBI [22]. In support of this notion, a recent study showed that the antigen presentation function of B cells is critical for the pathology of experimental autoimmune encephalitis (EAE), more so than auto-antibody production [34]. Thus, some consequences of TBI might depend upon efficient antigen processing and presentation by $\mathrm{B}$ cells.

The effector functions of B cells and T cells involve cytokines and chemokines. These small molecule compounds serve as chemotactic signaling proteins and immune modulators. Numerous studies have implicated a variety of chemokines and cytokines in the pathogenesis of TBI [32].
The interactions that govern the effects of cytokines are poorly understood in general, and even less-well understood in the context of TBI. In CD74 ${ }^{\text {Def }}$ mice, we observed significantly less IFNY and TNF $\alpha$ compared to WT controls following FPI. Elevations of both of these cytokines have been previously reported following various types of TBI [48-52], but the cellular source(s) of these cytokines and their functional significance are not well-defined. While some studies demonstrate that glial cells, including microglial cells are involved in the increase [53,54], the possibility that infiltrating immune cells, including macrophages, contribute to these increases cannot be ruled out. In support of this interpretation, we also observed decreased MIP1 $\beta$ (Figure 5) and RANTES, both of which are products of CD8+ T cells $[55,56]$, which were also reduced with CAP (Figure 1). Nevertheless, our data with CD74 ${ }^{\text {Def }}$ mice implicate antigen processing involving CD74 as a contributor to the elevation of these cytokines and chemokines after TBI.

While CCL2 is a well-described marker of inflammation after TBI $[57,58]$, and CAP reduced its expression in this study, less understood is the putative roles of IL15, IL13 and IL10, that were also reduced by CAP. Interestingly, a previous study using IL15 KO mice and IL15R KO mice in a facial axotomy model, found reduced $\mathrm{T}$ cell infiltration and increased MHCII + microglial cells [59]. Consistent with the potential involvement of macrophages/ microglial cells, IL13 has been previously shown to be involved in the elimination of activated microglial cells subsequent to an injury [60]. In our study, administration of CAP reduced the lesion size, which could reflect a reduction in the number of activated microglial cells responding to the injury, thereby decreasing the overall level of IL15 and IL-13. With regard to the CAP-dependent decrease in IL-10, previous studies have indicated that elevated IL-10 corresponds with a poor outcome for patients $[61,62]$. Therefore, the reduction in IL-10 observed following CAP treatment may be indicative of the decreased lesion size and thus an improved outcome.

\section{Conclusion}

The synthesis of CAP was based on the rationale that antagonism of CLIP would selectively target the transition between innate and adaptive immunity. While our results are consistent with this hypothesis, the consequences of CAP therapy resulting in neuroprotection require further examination to fully elucidate the precise mechanisms involved. Nonetheless, the results from the current study provide a foundation for targeting the switch between innate and adaptive immunity for the treatment of TBI. Moreover, our specific characterization of peripheral alterations to distinct subsets of immune cells may provide biomarkers for diagnosing and characterizing the degree of TBI severity, progression and/or recovery. 


\section{Additional files}

Additional file 1: Figure S1. Flow cytometric-gating strategy. Representative $5 \%$ contour plots of flow cytometric data to show gating strategy.

Additional file 2: Figure S2. The impact of CAP on the cell surface levels of CLIP and MHCII on B and $\gamma \delta$ T Cells. (A) The mean fluorescence intensity (MFI) of CLIP on the surface of B cells in the spleens of C57BL/6 mice 24 hours following sham surgery or FPI. (B) The number of CLIP ${ }^{+}$B cells $\left(\mathrm{B} 22 \mathrm{O}^{+} \mathrm{CLIP}^{+}\right)$. (C) The MFI of MHCll on the surface of $\mathrm{B}$ cells in the spleens of C57BL/6 mice 24 hours following sham surgery or FPI. (D) The $\mathrm{MFI}$ of CLIP on the surface of $\gamma \delta$ T cells in the spleens of C57BL/6 mice 24 hours following sham surgery or FPI. (E) The MFI of MHCII on the surface of $\gamma \delta T$ cells in the spleens of C57BL/6 mice 24 hours following sham surgery or FPI. Sham + CAP $(N=5) ; \mathrm{FPI}+$ vehicle $(\mathrm{N}=6)$.

\section{Competing interests}

RPT, SKR, JMK, and MKNR are scientific consultants for VG Life Sciences Inc., the license holder for CAP. RPT, MKNR, and LAS are all listed on a patent pending for CAP. Patent filed in the United States Patent and Trademark Office on September 9, 2013 under No. 61/875,670, bearing Attorney Docket No. V0223.70010US00.

\section{Authors' contributions}

RPT helped design experiments, performed experiments, analyzed data, and helped write the manuscript. SM helped design and perform experiments. JMK performed cytokine multiplex experiments. SKR, SKH, and HLM performed experiments. MKNR conceived the study, designed experiments, analyzed data, and helped write the manuscript. LAS helped conceive the study, designed experiments, analyzed data, and helped write the manuscript. All authors have read and approved the final manuscript.

\section{Authors' information}

RPT is a postdoctoral fellow, Department of Surgery, Texas A\&M University College of Medicine. SM is a research scientist, Department of Surgery, Texas A\&M University College of Medicine. JMK is a research assistant, Department of Surgery, Central Texas Veterans Health Care System, Texas A\&M University College of Medicine. SKR is an undergraduate student, Department of Anthropology, University of Texas, Austin. SKH is a research assistant, Department of Surgery, Baylor Scott and White Health. HLM is a research assistant, Department of Surgery, Texas A\&M University College of Medicine. MKNR is a Professor, Department of Surgery, Texas A\&M University College of Medicine, and Baylor Scott and White Health. LAS is an Assistant Professor, Department of Surgery, Central Texas Veterans Health Care System, Texas A\&M University College of Medicine.

\section{Acknowledgements}

This material is the result of work supported by the following: the Scott and White Healthcare Foundation (MKNR), the Wounded Warrior Fund (MKNR), VG Life Sciences Inc. (MKNR, RPT, LAS), Texas A\&M Health Sciences Center (MKNR, LAS) and the use of facilities at the Texas A\&M Health Science center (MKNR) and the Central Texas Veterans Health Care System, Temple, TX, USA (LAS). We are grateful to Evan W. Newell for his generous contributions to the design and generation of CAP. We gratefully acknowledge Glen Cryer for his support and his editorial assistance with the manuscript.

\section{Author details}

${ }^{1}$ Department of Surgery, Texas A\&M University Health Science Center, Temple, TX, USA. ${ }^{2}$ Central Texas Veterans Health Care System, Temple, TX, USA. ${ }^{3}$ Department of Anthropology, University of Texas, Austin, TX, USA. ${ }^{4}$ Department of Surgery, Baylor Scott and White Health, Temple, TX, USA. ${ }^{5}$ Texas A\&M Health Science Center, Baylor Scott and White Health, 702 SW. HK Dodgen Loop, MRB. Rm. 114B, Temple, TX 76504, USA.

\section{Received: 4 August 2014 Accepted: 11 September 2014}

\section{Published online: 20 October 2014}

\section{References}

1. Bazarian JJ, McClung J, Shah MN, Cheng YT, Flesher W, Kraus J (2005) Mild traumatic brain injury in the United States, 1998-2000. Brain Inj 19(2):85-91
2. Corrigan JD, Selassie AW, Orman JA (2010) The epidemiology of traumatic brain injury. J Head Trauma Rehabil 25(2):72-80, doi:10.1097/HTR.0b013 e3181ccc8b4

3. Hyder AA, Wunderlich CA, Puvanachandra P, Gururaj G, Kobusingye OC (2007) The impact of traumatic brain injuries: a global perspective. NeuroRehabilitation 22(5):341-353

4. Beschorner R, Schluesener HJ, Gozalan F, Meyermann R, Schwab JM (2002) Infiltrating CD14+ monocytes and expression of CD14 by activated parenchymal microglia/macrophages contribute to the pool of CD14+ cells in ischemic brain lesions. J Neuroimmunol 126(1-2):107-115

5. Das M, Mohapatra S, Mohapatra SS (2012) New perspectives on central and peripheral immune responses to acute traumatic brain injury. J Neuroinflammation 9:236, doi:10.1186/1742-2094-9-236

6. Finnie JW (2013) Neuroinflammation: beneficial and detrimental effects after traumatic brain injury. Inflammopharmacology 21(4):309-320, doi:10.1007/ s10787-012-0164-2

7. Clausen F, Lorant T, Lewen A, Hillered L (2007) T lymphocyte trafficking: a novel target for neuroprotection in traumatic brain injury. J Neurotrauma 24(8):1295-1307, doi:10.1089/neu.2006.0258

8. Medawar PB (1948) Immunity to homologous grafted skin; the fate of skin homografts transplanted to the brain, to subcutaneous tissue, and to the anterior chamber of the eye. Br J Exp Pathol 29(1):58-69

9. Butterfield TA, Best TM, Merrick MA (2006) The dual roles of neutrophils and macrophages in inflammation: a critical balance between tissue damage and repair. J Athl Train 41(4):457-465

10. Segel GB, Halterman MW, Lichtman MA (2011) The paradox of the neutrophil's role in tissue injury. J Leukoc Biol 89(3):359-372, doi:10.1189/ jlb.0910538

11. Hernandez-Ontiveros DG, Tajiri N, Acosta S, Giunta B, Tan J, Borlongan CV (2013) Microglia activation as a biomarker for traumatic brain injury. Front Neurol 4:30, doi:10.3389/fneur.2013.00030

12. Blum JS, Wearsch PA, Cresswell P (2013) Pathways of antigen processing. Annu Rev Immunol 31:443-473, doi:10.1146/annurev-immunol-032712095910

13. Allen PM, Babbitt BP, Unanue ER (1987) T-cell recognition of lysozyme: the biochemical basis of presentation. Immunol Rev 98:171-187

14. Barker CF, Billingham RE (1977) Immunologically privileged sites. Adv Immunol 25:1-54

15. Slavin AJ, Soos JM, Stuve O, Patarroyo JC, Weiner HL, Fontana A, Bikoff EK, Zamvil SS (2001) Requirement for endocytic antigen processing and influence of invariant chain and $\mathrm{H}-2 \mathrm{M}$ deficiencies in CNS autoimmunity. J Clin Invest 108(8):1133-1139, doi:10.1172/JCl13360

16. Ling C, Sandor M, Suresh M, Fabry Z (2006) Traumatic injury and the presence of antigen differentially contribute to T-cell recruitment in the CNS. J Neurosci 26(3):731-741, doi:10.1523/JNEUROSCI.3502-05.2006

17. Schori H, Lantner F, Shachar I, Schwartz M (2002) Severe immunodeficiency has opposite effects on neuronal survival in glutamate-susceptible and -resistant mice: adverse effect of B cells. J Immunol 169(6):2861-2865

18. Schori H, Shechter R, Shachar I, Schwartz M (2007) Genetic manipulation of CD74 in mouse strains of different backgrounds can result in opposite responses to central nervous system injury. J Immunol 178(1):163-171

19. Schori H, Yoles E, Wheeler LA, Raveh T, Kimchi A, Schwartz M (2002) Immune-related mechanisms participating in resistance and susceptibility to glutamate toxicity. Eur J Neurosci 16(4):557-564

20. Babbitt BP, Allen PM, Matsueda G, Haber E, Unanue ER (1985) Binding of immunogenic peptides to la histocompatibility molecules. Nature 317 (6035):359-361

21. Cheng L, Cui Y, Shao H, Han G, Zhu L, Huang Y, O'Brien RL, Born WK, Kaplan HJ, Sun D (2008) Mouse gammadelta T cells are capable of expressing MHC class II molecules, and of functioning as antigen-presenting cells. J Neuroimmunol 203(1):3-11, doi:10.1016/j.jneuroim.2008.06.007

22. Lanzavecchia A (1987) Antigen uptake and accumulation in antigen-specific B cells. Immunol Rev 99:39-51

23. Denzin LK, Cresswell P (1995) HLA-DM induces CLIP dissociation from MHC class II alpha beta dimers and facilitates peptide loading. Cell 82(1):155-165

24. Buus S, Sette A, Colon SM, Jenis DM, Grey HM (1986) Isolation and characterization of antigen-la complexes involved in T cell recognition. Cell 47(6):1071-1077

25. Newell MK, Tobin RP, Cabrera JH, Sorensen MB, Huckstep A, Villalobos-Menuey EM, Burnett M, McCrea E, Harvey CP, Buddiga A, Bar-Or A, Freedman MS, Nalbantoglu J, Arbour N, Zamvil SS, Antel JP 
(2010) TLR-mediated B cell activation results in ectopic CLIP expression that promotes B cell-dependent inflammation. J Leukoc Biol 88(4):779-789, doi:jb.0410237 [pii] 10.1189/jlb.0410237

26. Mukherjee S, Zeitouni S, Cavarsan CF, Shapiro LA (2013) Increased seizure susceptibility in mice 30 days after fluid percussion injury. Front Neurol 4:28, doi:10.3389/fneur.2013.00028

27. Davidson WF, Parish CR (1975) A procedure for removing red cells and dead cells from lymphoid cell suspensions. J Immunol Methods 7(2-3):291-300

28. Amini M, Nahrevanian H, Farahmand M (2008) Pathogenicity variations of susceptibility and resistance to Leishmania major MRHO/R/75/ER Strain in BALB/C and C57BL/6 mice. Iran J Parasitol 3(4):51-59

29. Oporto VG, Fuentes R, Borie E, Del Sol M, Orsi IA, Engelke W (2014) Radiographical and clinical evaluation of critical size defects in rabbit calvaria filled with allograft and autograft: a pilot study. Int J Clin Exp Med 7(7):1669-1675

30. Franklin KBJ, Paxinos G (1997) The Mouse Brain in Stereotaxic Coordinates. Academic, San Diego

31. Collins RA, Werling D, Duggan SE, Bland AP, Parsons KR, Howard CJ (1998) Gammadelta T cells present antigen to CD4+ alphabeta T cells. J Leukoc Biol 63(6):707-714

32. Mukherjee S, Katki K, Arisi GM, Foresti ML, Shapiro LA (2011) Early TBl-induced cytokine alterations are similarly detected by two distinct methods of multiplex assay. Front Mol Neurosci 4:21, doi:10.3389/fnmol.2011.00021

33. Moalem G, Leibowitz-Amit R, Yoles E, Mor F, Cohen IR, Schwartz M (1999) Autoimmune $T$ cells protect neurons from secondary degeneration after central nervous system axotomy. Nat Med 5(1):49-55, doi:10.1038/4734

34. Molnarfi N, Schulze-Topphoff U, Weber MS, Patarroyo JC, Prod'homme T, Varrin-Doyer M, Shetty A, Linington C, Slavin AJ, Hidalgo J, Jenne DE, Wekerle H, Sobel RA, Bernard CC, Shlomchik MJ, Zamvil SS (2013) MHC class II-dependent B cell APC function is required for induction of CNS autoimmunity independent of myelin-specific antibodies. J Exp Med 210 (13):2921-2937, doi:10.1084/jem.20130699

35. Leng L, Metz CN, Fang Y, Xu J, Donnelly S, Baugh J, Delohery T, Chen Y, Mitchell RA, Bucala R (2003) MIF signal transduction initiated by binding to CD74. J Exp Med 197(11):1467-1476, doi:10.1084/jem.20030286

36. Liao H, Bucala R, Mitchell RA (2003) Adhesion-dependent signaling by macrophage migration inhibitory factor (MIF). J Biol Chem 278(1):76-81, doi:10.1074/jbc.M208820200

37. Meyer-Siegler KL, Iczkowski KA, Leng L, Bucala R, Vera PL (2006) Inhibition of macrophage migration inhibitory factor or its receptor (CD74) attenuates growth and invasion of DU-145 prostate cancer cells. J Immunol 177 (12):8730-8739

38. Shi X, Leng L, Wang T, Wang W, Du X, Li J, McDonald C, Chen Z, Murphy JW, Lolis E, Noble P, Knudson W, Bucala R (2006) CD44 is the signaling component of the macrophage migration inhibitory factor-CD74 receptor complex. Immunity 25(4):595-606, doi:10.1016/j.immuni.2006.08.020

39. Bittner $S$, Ruck T, Schuhmann MK, Herrmann AM, Moha ou Maati H, Bobak N, Gobel K, Langhauser F, Stegner D, Ehling P, Borsotto M, Pape HC, Nieswandt B, Kleinschnitz C, Heurteaux C, Galla HJ, Budde T, Wiendl H, Meuth SG (2013) Endothelial TWIK-related potassium channel-1 (TREK1) regulates immune-cell trafficking into the CNS. Nat Med 19(9):1161-1165, doi:10.1038/nm.3303

40. Li P, Gan Y, Sun BL, Zhang F, Lu B, Gao Y, Liang W, Thomson AW, Chen J, Hu X (2013) Adoptive regulatory T-cell therapy protects against cerebral ischemia. Ann Neurol 74(3):458-471, doi:10.1002/ana.23815

41. Li P, Mao L, Zhou G, Leak RK, Sun BL, Chen J, Hu X (2013) Adoptive regulatory T-cell therapy preserves systemic immune homeostasis after cerebral ischemia. Stroke 44(12):3509-3515, doi:10.1161/STROKEAHA.113.002637

42. Liesz A, Zhou W, Na SY, Hammerling GJ, Garbi N, Karcher S, Mracsko E, Backs J, Rivest S, Veltkamp R (2013) Boosting regulatory T cells limits neuroinflammation in permanent cortical stroke. J Neurosci 33(44):17350-17362, doi:10.1523/JNEUROSCI.4901-12.2013

43. Zhou W, Liesz A, Bauer H, Sommer C, Lahrmann B, Valous N, Grabe N, Veltkamp R (2013) Postischemic brain infiltration of leukocyte subpopulations differs among murine permanent and transient focal cerebral ischemia models. Brain Pathol 23(1):34-44, doi:10.1111/j.1750-3639.2012.00614.x

44. Haribhai D, Williams JB, Jia S, Nickerson D, Schmitt EG, Edwards B, Ziegelbauer J, Yassai M, Li SH, Relland LM, Wise PM, Chen A, Zheng YQ, Simpson PM, Gorski J, Salzman NH, Hessner MJ, Chatila TA, Williams CB (2011) A requisite role for induced regulatory $T$ cells in tolerance based on expanding antigen receptor diversity. Immunity 35(1):109-122, doi:10.1016/j. immuni.2011.03.029
45. Weiss JM, Bilate AM, Gobert M, Ding Y, de Lafaille MA C, Parkhurst CN, Xiong H, Dolpady J, Frey AB, Ruocco MG, Yang Y, Floess S, Huehn J, Oh S, Li MO, Niec RE, Rudensky AY, Dustin ML, Littman DR, Lafaille JJ (2012) Neuropilin 1 is expressed on thymus-derived natural regulatory T cells, but not mucosa-generated induced Foxp3+ T reg cells. J Exp Med 209 (10):1723-1742, S1721. doi:10.1084/jem.20120914

46. Freedman MS, Ruiis TC, Selin LK, Antel JP (1991) Peripheral blood gamma-delta $T$ cells lyse fresh human brain-derived oligodendrocytes. Ann Neurol 30 (6):794-800, doi:10.1002/ana.410300608

47. Zeine R, Pon R, Ladiwala U, Antel JP, Filion LG, Freedman MS (1998) Mechanism of gammadelta T cell-induced human oligodendrocyte cytotoxicity: relevance to multiple sclerosis. J Neuroimmunol 87(1-2):49-61

48. Cho HJ, Sajja VS, Vandevord PJ, Lee YW (2013) Blast induces oxidative stress, inflammation, neuronal loss and subsequent short-term memory impairment in rats. Neuroscience 253:9-20, doi:10.1016/..neuroscience.2013.08.037

49. Fan L, Young PR, Barone FC, Feuerstein GZ, Smith DH, McIntosh TK (1996) Experimental brain injury induces differential expression of tumor necrosis factor-alpha mRNA in the CNS. Brain Res Mol Brain Res 36(2):287-291

50. Holmin S, Hojeberg B (2004) In situ detection of intracerebral cytokine expression after human brain contusion. Neurosci Lett 369(2):108-114, doi:10.1016/.neulet.2004.07.044

51. Morganti-Kossman MC, Lenzlinger PM, Hans V, Stahel P, Csuka E, Ammann E, Stocker R, Trentz O, Kossmann T (1997) Production of cytokines following brain injury: beneficial and deleterious for the damaged tissue. Mol Psychiatry 2(2):133-136

52. Shohami E, Novikov M, Bass R, Yamin A, Gallily R (1994) Closed head injury triggers early production of TNF alpha and IL-6 by brain tissue. J Cereb Blood Flow Metab 14(4):615-619, doi:10.1038/jcbfm.1994.76

53. Cheong CU, Chang CP, Chao CM, Cheng BC, Yang CZ, Chio CC (2013) Etanercept attenuates traumatic brain injury in rats by reducing brain TNF- alpha contents and by stimulating newly formed neurogenesis. Mediat Inflamm 2013:620837, doi:10.1155/2013/620837

54. Chio CC, Chang $\mathrm{CH}$, Wang $\mathrm{CC}$, Cheong $\mathrm{CU}$, Chao $\mathrm{CM}$, Cheng $\mathrm{BC}$, Yang $\mathrm{CZ}$, Chang CP (2013) Etanercept attenuates traumatic brain injury in rats by reducing early microglial expression of tumor necrosis factor-alpha. BMC Neurosci 14:33, doi:10.1186/1471-2202-14-33

55. Cocchi F, DeVico AL, Garzino-Demo A, Arya SK, Gallo RC, Lusso P (1995) Identification of RANTES, MIP-1 alpha, and MIP-1 beta as the major HIV-suppressive factors produced by CD8+ T cells. Science 270(5243):1811-1815

56. Song A, Nikolcheva T, Krensky AM (2000) Transcriptional regulation of RANTES expression in T lymphocytes. Immunol Rev 177:236-245

57. Liu S, Zhang L, Wu Q, Wu Q, Wang T (2013) Chemokine CCL2 induces apoptosis in cortex following traumatic brain injury. J Mol Neurosci 51 (3):1021-1029, doi:10.1007/s12031-013-0091-8

58. Semple BD, Bye N, Rancan M, Ziebell JM, Morganti-Kossmann MC (2010) Role of CCL2 (MCP-1) in traumatic brain injury (TBI): evidence from severe TBI patients and CCL2-/- mice. J Cereb Blood Flow Metab 30(4):769-782, doi:10.1038/jcbfm.2009.262

59. Huang Z, Ha GK, Petitto JM (2007) IL-15 and IL-15R alpha gene deletion: effects on T lymphocyte trafficking and the microglial and neuronal responses to facial nerve axotomy. Neurosci Lett 417(2):160-164, doi:10.1016/..neulet.2007.01.086

60. Yang MS, Park EJ, Sohn S, Kwon HJ, Shin WH, Pyo HK, Jin B, Choi KS, Jou I, Joe EH (2002) Interleukin-13 and -4 induce death of activated microglia. Glia 38(4):273-280, doi:10.1002/glia.10057

61. Ferreira LC, Regner A, Miotto KD, Moura S, Ikuta N, Vargas AE, Chies JA, Simon D (2014) Increased levels of interleukin-6, -8 and -10 are associated with fatal outcome following severe traumatic brain injury. Brain Inj 28 (10):1311-1316, doi:10.3109/02699052.2014.916818

62. Schneider Soares FM, Menezes de Souza N, Liborio Schwarzbold M, Paim Diaz A, Costa Nunes J, Hohl A, Abreu N, da Silva P, Vieira J, Lisboa de Souza R, More Bertotti M, Schoder Prediger RD, Neves Linhares M, Bafica A, Walz R (2012) Interleukin-10 is an independent biomarker of severe traumatic brain injury prognosis. Neuroimmunomodulation 19(6):377-385, doi: 10.1159/000342141

\section{doi:10.1186/s40478-014-0143-5}

Cite this article as: Tobin et al:: Traumatic brain injury causes selective, CD74-dependent peripheral lymphocyte activation that exacerbates neurodegeneration. Acta Neuropathologica Communications 2014 2:143. 